\title{
The center magnetic vortex and its influence on physical quantities in the gluon plasma
}

\section{Takuya Saito*}

Research and Education Faculty, Natural Sciences Cluster, Sciences Unit, Integrated Information Center, Kochi University, Akebono-cho, Kochi, 780-8520, Japan E-mail: tsaitou@kochi-u.ac.jp

\begin{abstract}
We show the evidence that magnetic degrees of freedom are so important in the deconfinement phase by a lattice numerical simulation in magnetic center vortices. The equation of state of the gluon plasma has a large contribution obtained from the vortices after the phase transition.
\end{abstract}

The XXIX International Symposium on Lattice Field Theory - Lattice 2011

July 10-16, 2011

Squaw Valley, Lake Tahoe, California

\footnotetext{
* Speaker.
} 


\section{Introduction}

The heavy-ion collision experiment (RHIC and LHC) produces the new matter that the system temperature exceeds the critical temperature $\left(T_{c}\right)$ of the transition from the hadron to the quarkgluon plasma (QGP) phases. At first, a study of the QGP physics starts from the assumption that the deconfining gluons and quarks above $T_{c}$ are freely moving, so that the conventional perturbation theory is valid in the deconfinement phase. However, it is found, through the many phenomenological analyses and lattice computations, that the discovered QGP matter is not a weakly-coupling plasma but strongly-interacting QGP (sQGP) matters. Therefore, it is still an unsolved problem what mechanism in quantum chromodynamics (QCD) in the hot phase causes such strong interactions.

One of the interesting ideas to describe such a peculiar sQGP is to focus on a singularity coming from the magnetic degrees of freedom [1]. In the thermal QCD theory, the magnetic gluon propagators in the zero-momentum limit diverges if its effective mass vanishes and then the perturbation completely fails. Therefore, the non-zero magnetic mass is required an infrared regulator. The lattice results show that the magnetic mass is not zero [2,3]. Moreover, a spatial Wilson-loop and a color-Coulomb instantaneous potentials in the deconfining phase give also a non-zero string tension, which is in proportional to the temperature dependence of the magnetic mass $[4,5]$.

In many cases of the non-Abelian gauge theory above $T_{c}$, the magnetic and/or spatial components are linked to some (infrared-singular) confining objects. In the point of view of the topological defect on the lattice, the magnetic monopole and/or vortex play an important role for a spatial (magnetic) confinement, while the temporal (electric) degrees of freedom is deconfing (because this is confirmed by the behavior of the time-like Polyakov line near $\left.T_{c}[6,7]\right)$. The center vortex mechanism also holds well over hot Yang-Mills theory[8]; as a result, the hot-glue energy-momentum tensor is largely affected [9]. Those non-vanishing contribution from the hot vortices is responsible for the magnetic singularity of the gluon propagator [10]. In the similar way, the importance of the thermal monopole (connected by vortices) has been discussed [11].

We here study the equation of state (EOS) of the gluon plasma in terms of the center vortex degrees of freedom by using an $S U(2)$ quenched-lattice calculation. Removing center vortices from the original lattice enables us to investigate the effect of them on the gluon EOS, and the change of the correlators of the transport coefficient, particular for shear viscosity, which is observed to be nearly zero, meaning that QGP is almost perfect fluid.

\section{Formulations}

\subsection{Center vortex projection}

We here use direct maximal center projection (MCP) [12], so as to identify center vortex on the lattice. This method is implemented by realizing the following condition to updated link variables,

$$
\text { Maximize } R=\frac{1}{V N_{t}} \sum_{x} \operatorname{Tr}\left[U_{\mu}(x)\right]^{2}
$$


so that a center field function on $S U(2)$ can be defined as

$$
Z_{\mu}(x)=\operatorname{sgn} \operatorname{Tr}\left[U_{\mu}(x)\right] \in \mathbb{Z}_{2} .
$$

The plaquette value forming from four links is plus if all the corresponding $Z_{\mu}(x)$ is plus, while if the plaquette includes a minus link an odd times then it becomes also minus. This minus plaquette is a non-trivial negative energy of gluon fields; therefore, the center vortex pierces in this square region

In addition, one can remove the center vortex contribution from the original lattice configuration by multiplying $Z_{\mu}(x)$ to the center projected link as [13]

$$
U_{\mu}^{\prime}(x)=Z_{\mu} U_{\mu}(x)
$$

This enables us to obtain a new non-confing theory consisting of these new links. $Z_{\mu}$ carries nonperturbative information upon the color confinement and chiral symmetry breaking $(\chi \mathrm{SB})$. The lattice simulations show that non-zero string tension as well as the chiral condensation are lost after the center removal $[12,13]$.

\subsection{Measurements}

The equation of state of the gluon plasma on the lattice can be calculated via the plaquette expectation value with the lattice coupling $\beta$ as

$$
\left.\frac{p}{T^{4}}\right|_{\beta_{0}} ^{\beta}=\int_{\beta_{0}}^{\beta} d \beta^{\prime} \Delta S
$$

where $T=1 /\left(N_{t} a\right)$ and

$$
\Delta S=N_{t}^{4}\left(\langle S\rangle_{T}-\langle S\rangle_{0}\right),
$$

and $\left\langle S_{T}>\right.$ and $<S_{0}>$ are the expectation values on the anti-symmetric and symmetric lattices $[14,15]$.

Moreover, in order to investigate the critical phenomena one can use the expectation values of the Polyakov line which is defined by

$$
P(\vec{x})=\frac{1}{2} \operatorname{Tr} \prod_{t=0}^{t=N_{t}} U_{0}(\vec{x}, t)
$$

This is usually considered as an order parameter on the confinement/deconfinement phase transition.

\section{Numerical results}

We here carry out the Monte Carlo simulation with standard plaquette action of $S U(2)$ gauge theory. We apply the MCP to all gauge configurations updated, and subsequently prepare new configurations after the removal of center vortices, so as to compare with the measurements calculated by the normal lattice ones.

In Fig.1 we plot the $\Delta S$ values of a function of $\beta$ for the vortex removal and normal lattice configurations. For the normal ones, after the phase transition (above $\beta=2.30$ ), $\Delta S$ rapidly goes 
up and as the $\beta$ goes higher it asymptotically approaches zero axis. This behavior has been well known by a lot of the previous calculations. On the other hand, if one removes vortex degrees of freedom from the original lattice, $\Delta S$ becomes larger than the normal. This means that in addition to the electric degrees of freedom after the phase transition, the magnetic (confining) modes are also released; note that even in the deconfining phase, the spatial gluon components still are confing. Thus the center-removing lattice configuration yields large contribution to the equation of state.
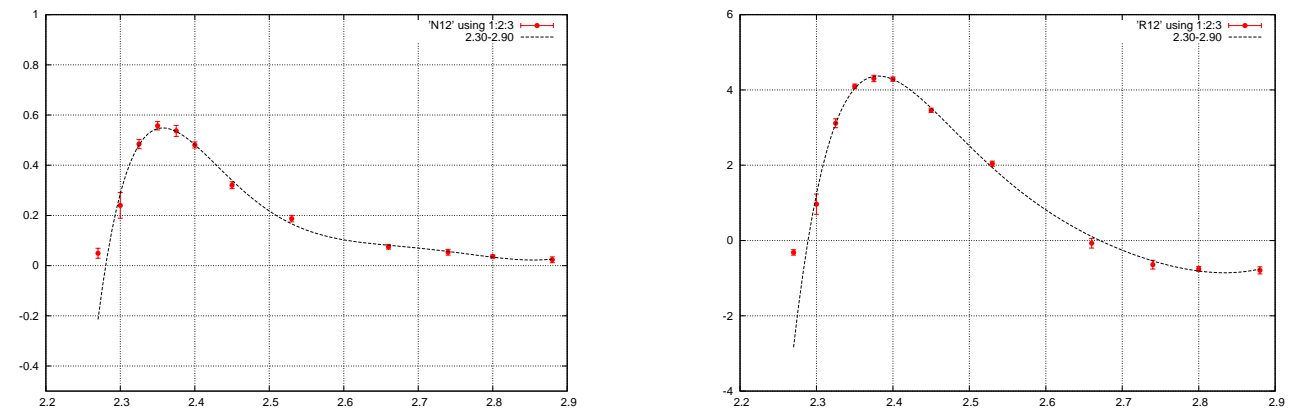

Figure 1: $\Delta S$ as a function of $\beta$ on the removal (of center vortices) and normal configurations. This lattice size is $16^{3} \times 4$ and the critical $\beta_{c} \approx 2.3$. The dashed-lines are numerically determined by using a polynomial of $\beta$.

In Fig. 2 we show a graph of the pressures $p / T^{4}$ calculated from the normal and center vortex removal configurations. This has been done by integrating the fitting functions of $\beta$ plotting on the Fig. 1. One sees that the equation of state of center-vortex-free has larger contributions than the normal one. This means that the magnetic degrees of freedom (released after the center removal) are important modes even in the deconfining phase.

Besides, in the higher $\beta$ regions, $\Delta S$ becomes a minus; this contributes negative to EOS. Therefore one needs to do an extensive lattice simulation to investigate such volume size dependence or higher temperature limit.

In Fig.3 we plot the numerical data of the expectation values of the electric-Poylakov line as a function of the lattice coupling $\beta$. In the confinement phase the Poylakov line becomes non-zero if removing vortices from the confining lattice; namely, it is a non-confining theory. However, the both data between the removal and normal lattices approach each other slowly as the beta increases. At high beta (about over $\beta=2.6$ which is approximately $T / T_{c}=2.0-3.0$ ) the remnant after removing center vortices seems to vanishes. This may means that the electric modes are completely free objects for such temperature regions; on the other side, near $T_{c}$, the electric gluons are also affected strongly due to the magnetic degrees freedom resulting from magnetic vortices.

\section{Summary}

We have investigated the effect of the center magnetic vortices in the quark-gluon plasma by using the $S U$ (2) lattice gauge simulations. The center vortex is a topological defect of $S U(N)$ lattice theory and is responsible for the color confinement and chiral symmetry breaking. We calculated the equation of state of QGP after the phase transition and found very large contributions from the 


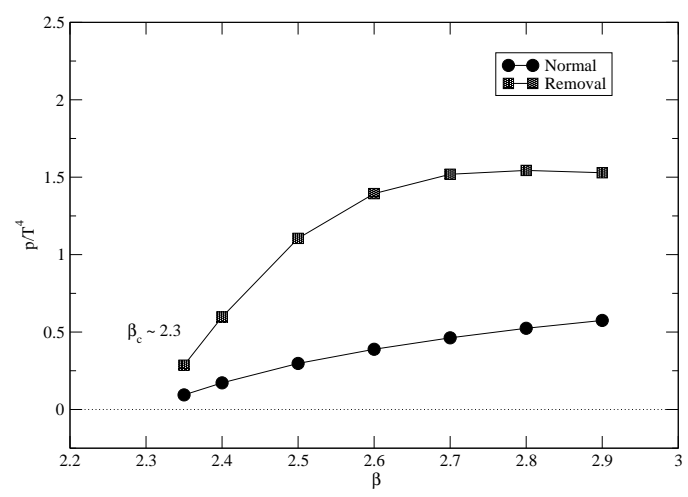

Figure 2: The comparison of the pressures $\left(p / T^{4}\right)$ versus $\beta$ upon the normal and vortex removed configurations.

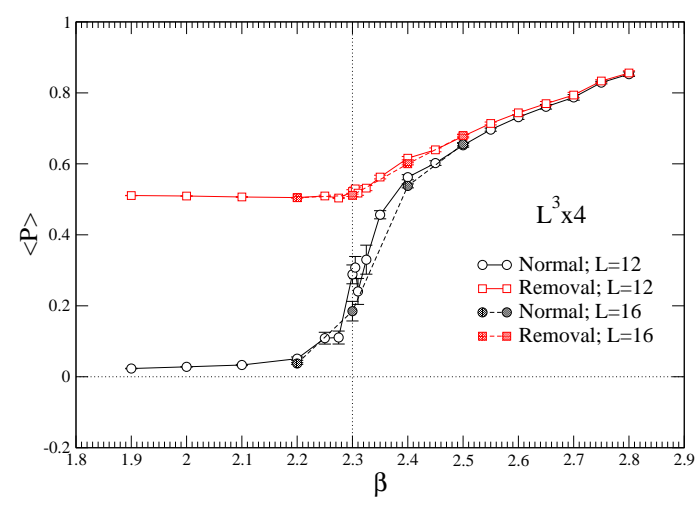

Figure 3: The expectation values of the electric Polyakov line vs. $\beta$ upon the normal and vortex removed configurations.

center vortices. This means that the magnetic degrees of freedom are still singular objects even in the deconfining phase. This phenomenon emerges as a magnetic mass of the gluon propagators in the thermal medium. The similar behavior is also given by plotting the Poylakov line expectation values versus $\beta$; it is found that from the confining phase to the deconfining phase, center vortices (magnetic modes) play an important role for understanding the physics of QGP.

Our calculations presented here are preliminary numerical results, and so as to obtain a final conclusion one needs to do more extensive simulations on the larger lattice and high temperature regions. 


\section{References}

[1] A.D. Linde, Phys. Lett. B96, 289.

[2] A. Nakamura, T. Saito, S. Sakai, Phys.Rev. D69 (2004) 014506; A. Nakamura, I. Pushkina, T. Saito, S. Sakai, Phys.Lett. B549 (2002) 133-138

[3] Y. Maezawa, S. Aoki, S. Ejiri, T. Hatsuda, N. Ishii, K. Kanaya, N. Ukita, T. Umeda (WHOT-QCD Collaboration), Phys.Rev.D81:091501,2010.

[4] A. Nakamura and T. Saito, Prog. Theor. Phys. 115, 189 (2006).

[5] Y. Nakagawa, A. Nakamura, T. Saito, H. Toki and D. Zwanziger, Phys. Rev. D 73, 094504 (2006).

[6] A. Nakamura and T. Saito, Prog.Theor.Phys. 112 (2004) 183-188.

[7] A. Nakamura and T. Saito, Prog.Theor.Phys. 111 (2004) 733-743.

[8] M.N. Chernodub, V.I. Zakharov, Phys. Rev. Lett. 98,082002,2007

[9] in equation of state of Yang-Mills plasma" M.N. Chernodub, Atsushi Nakamura, V.I. Zakharov, Phys.Rev.D78,074021,2008.

[10] M.N. Chernodub, Y. Nakagawa, A. Nakamura, T. Saito, V. I. Zakharov, Phys. Rev. D83,114501,2011.

[11] J. Liao and E. Shuryak, Phys.Rev.Lett.101,162302,2008; J. Liao, E. Shuryak, J. Phys. G35,104058,2008

[12] L. Del Debbio, M. Faber, J. Giedt, J. Greensite, S. Olejnik, Phys.Rev.D58,094501,1998

[13] Ph. de Forcrand, M. D’Elia, Phys. Rev. Lett. 82 (1999) 4582

[14] G. Boyd, et al., Nucl. Phys. B469 (1996) 410-444.

[15] M. Okamoto, et al., Phys. Rev. D V60 (1999) 094510. 